\title{
Mechanism of tumour vascularization in experimental lung metastases
}

\author{
Vanessza Szabo,' Edina Bugyik,' Katalin Dezso,' Nora Ecker,' Peter Nagy,' Jozsef Timar,2,3 Jozsef Tovari,4,5 Viktoria \\ Laszlo, ${ }^{6}$ Victoria L Bridgeman,7 Elaine Wan,7 Sophia Frentzas,7 Peter B Vermeulen,,8 Andrew R Reynolds, ${ }^{7 *, \dagger}$ \\ Balazs Dome $5,6,9,10, \uparrow * *$ and Sandor Paku ${ }^{1,2 *, \uparrow}$ \\ Ist Department of Pathology and Experimental Cancer Research, Semmelweis University, Budapest, Hungary \\ 2 Tumor Progression Research Group, Hungarian Academy of Sciences-Semmelweis University, Budapest, Hungary \\ 3 2nd Department of Pathology, Semmelweis University, Budapest, Hungary \\ 4 Department of Experimental Pharmacology, National Institute of Oncology, Budapest, Hungary \\ 5 Department of Thoracic Surgery, Semmelweis University-National Institute of Oncology, Budapest, Hungary \\ 6 Department of Thoracic Surgery, Medical University of Vienna, Austria \\ 7 Tumour Biology Team, Breakthrough Breast Cancer Research Centre, The Institute of Cancer Research, London, UK \\ 8 Translational Cancer Research Unit, GZA Hospitals Sint-Augustinus, Antwerp, Belgium \\ 9 National Koranyi Institute of Pulmonology, Budapest, Hungary \\ 10 Department of Biomedical Imaging and Image-guided Therapy, Medical University of Vienna, Austria
}

\begin{abstract}
* Correspondence to: Andrew R Reynolds, PhD, Breakthrough Breast Cancer Research Centre, The Institute of Cancer Research, 237 Fulham Road, London SW3 6JB, UK. E-mail: andrew.reynolds@icr.ac.uk

Or Balazs Dome, MD, PhD, Department of Thoracic Surgery, Medical University of Vienna, Waehringer Guertel 18-20, A- 1090 Vienna, Austria. E-mail: balazs.dome@meduniwien.ac.at

Or Sándor Paku, PhD, Ist Institute of Pathology and Experimental Cancer Research, Semmelweis University ÜII'"oi út 26, H-1085, Budapest, Hungary. E-mail: paku@korbl.sote.hu
\end{abstract}

${ }^{\dagger} A R R, B D$, and SP are co-senior and co-corresponding authors to this study.

\begin{abstract}
The appearance of lung metastases is associated with poor outcome and the management of patients with secondary pulmonary tumours remains a clinical challenge. We examined the vascularization process of lung metastasis in six different preclinical models and found that the tumours incorporated the pre-existing alveolar capillaries (ie vessel co-option). During the initial phase of vessel co-option, the incorporated capillaries were still sheathed by pneumocytes, but these incorporated vessels subsequently underwent different fates dependent on the model. In five of the models examined (B16, HT1080, HT25, C26, and MAT B-III), the tumour cells gradually stripped the pneumocytes from the vessels. These dissected pneumocytes underwent fragmentation, but the incorporated microvessels survived. In the sixth model (C38), the tumour cells failed to invade the alveolar walls. Instead, they induced the development of vascularized desmoplastic tissue columns. Finally, we examined the process of arterialization in lung metastases and found that they became arterialized when their diameter grew to exceed $5 \mathrm{~mm}$. In conclusion, our data show that lung metastases can vascularize by co-opting the pulmonary microvasculature. This is likely to have important clinical implications, especially with respect to anti-angiogenic therapies.

Copyright $@ 2014$ Pathological Society of Great Britain and Ireland. Published by John Wiley \& Sons, Ltd.
\end{abstract}

Keywords: lung metastasis; vessel co-option; arterialization; angiogenesis

Received 20 August 2014; Revised 3 October 2014; Accepted 13 October 2014

No conflicts of interest were declared.

\section{Introduction}

Metastasis - the dissemination of malignant cells from a primary tumour to distant sites - is the main cause of death in many cancers, including breast, colorectal, melanoma, pancreatic, renal, and ovarian cancer. The lung is a frequently involved site in metastatic disease [1], with metastases found in $20-54 \%$ of extrathoracic tumours [2-4]. The only method that so far has demonstrated a real potential to treat patients with lung metastasis is surgical metastasectomy [5]. A report summarizing the data of more than 5000 patients identified resectability, the number of metastases, disease-free interval after surgery, and histology as important prognostic factors for lung metastasectomy [6]. Unfortunately, however, most patients with lung metastasis are not eligible for surgery and are managed with systemic or locoregional chemo- and/or targeted therapies, the efficacy of which is still modest [7-9]. 
Access to a sufficient blood supply is considered essential for the growth of metastases [10]. The study of the tumour vasculature in metastases is important for several reasons. Firstly, the effective delivery of any given anti-cancer drug is dependent on the presence of an efficient tumoural blood supply and is influenced by heterogeneities in the blood supply between different intratumoural areas [11]. Secondly, conventional drugs designed to target the tumour vasculature (ie anti-angiogenic agents) have shown mixed and often disappointing results in the treatment of metastatic cancer [12-15]. Therefore, a better understanding of the tumour vascularization process in metastases is critical for the development of more successful therapeutic strategies for treating metastatic disease.

Based on early observations on tumours growing in artificial locations such as the subcutaneous space, tumour vascularization had been long considered to require sprouting angiogenesis [16-18]. The first description of a so-called alternative (ie non-sprouting) tumour vascularization mechanism [10] dates back to the mid-1990s, when Pezzella et al published their groundbreaking work on non-angiogenic primary [19] and metastatic [20] lung tumours that grow by exploiting the pre-existing lung vasculature. These studies in human pathology provided evidence that both non-small cell lung cancer (NSCLC) [19] and pulmonary metastases of breast cancer [20] can develop and progress in the absence of neo-angiogenesis by co-opting the alveolar capillaries. Subsequent human studies from Pezzella and co-workers [21,22] and other groups $[23,24]$ have confirmed the phenomenon of vessel co-option in non-angiogenic primary and secondary lung tumours and, moreover, in various other primary and metastatic human malignancies (reviewed in ref 25). Nevertheless, the exact mechanism of vessel co-option in lung metastasis has been largely unexplored.

Here, by using confocal microscopy, electron microscopy, and a two-colour corrosion casting technique, we studied the tumour vascularization process in six different preclinical models of lung metastasis. Our results provide the first direct experimental evidence that pulmonary metastases can vascularize via co-opting the pre-existing pulmonary vasculature. Our study also presents the detailed mechanism of this process and, moreover, demonstrates that lung metastases develop a bronchial (ie arterial) blood supply during their growth. These findings are likely to have important implications with respect to the treatment of lung metastasis.

\section{Materials and methods}

\section{Tumour cell lines and experimental lung metastases}

The HT1080 human fibrosarcoma, HT25 human colon carcinoma, C26 murine colon carcinoma, MAT-B-III rat mammary carcinoma, and B16 mouse melanoma cell lines were cultured in RPMI-1640 medium supplemented with $10 \%$ fetal bovine serum (catalogue
Nos R8758 and F4135, respectively; Sigma-Aldrich, St Louis, MO, USA). C38 murine colon carcinoma cells were maintained by serial subcutaneous transplantations in $\mathrm{C} 57 \mathrm{Bl} / 6$ mice. A detailed description of the pulmonary metastasis models may be found in the Supplementary materials and methods.

Immunofluorescence analysis, tumour and endothelial cell proliferation, and electron microscopy

The immunofluorescence methods and electron microscopic analysis of lung metastases performed on B16 and HT1080 tumour lines may be found in the Supplementary materials and methods and Supplementary Table 1.

\section{Vascular corrosion casting: determination of the percentage of arterial metastases and the size of the metastases}

In contrast to the mouse lung, which does not have a functional intraparenchymal bronchial arterial vasculature, the existence of such a circulatory system in the rat lungs is proven [26,27]. Consequently, these experiments were carried out in rats carrying pulmonary metastases of MAT-B-III cells. To determine the size of the metastases and to investigate the origin of their blood supply, we used a two-colour corrosion casting procedure described previously [28] and presented in the Supplementary materials and methods.

\section{Results}

\section{Preservation of alveolar architecture in the} peripheral regions of lung metastases that is indicative of vessel co-option

We studied the process of tumour vascularization in six different models of experimental lung metastasis. Experimental lung metastases were generated by injecting HT1080, HT25, B16 or C26 cells intravenously into mice or, in the case of MAT-B-III, into mice or rats. Experimental lung metastases of C38 cells were generated using a spontaneous model of lung metastasis involving the injection of tumour cells into the footpad of the hind leg. The size of the metastatic lung nodules that we studied ranged from $100 \mu \mathrm{m}$ up to several millimetres. Larger tumours ( $>5 \mathrm{~mm}$ in diameter) were observed in rats injected with MAT-B-III. The mechanism of vascularization that we observed was independent of tumour size.

After extravasation and forming small interstitial colonies (ie growth of tumour cells within the alveolar walls), tumour cells proceeded to enter the alveolar air spaces. The expansion from one alveolar space to another was the default pattern for all of the tumour cell lines studied (Figures 1A and 1B). This was evident because the normal structure of the alveolar walls, which 

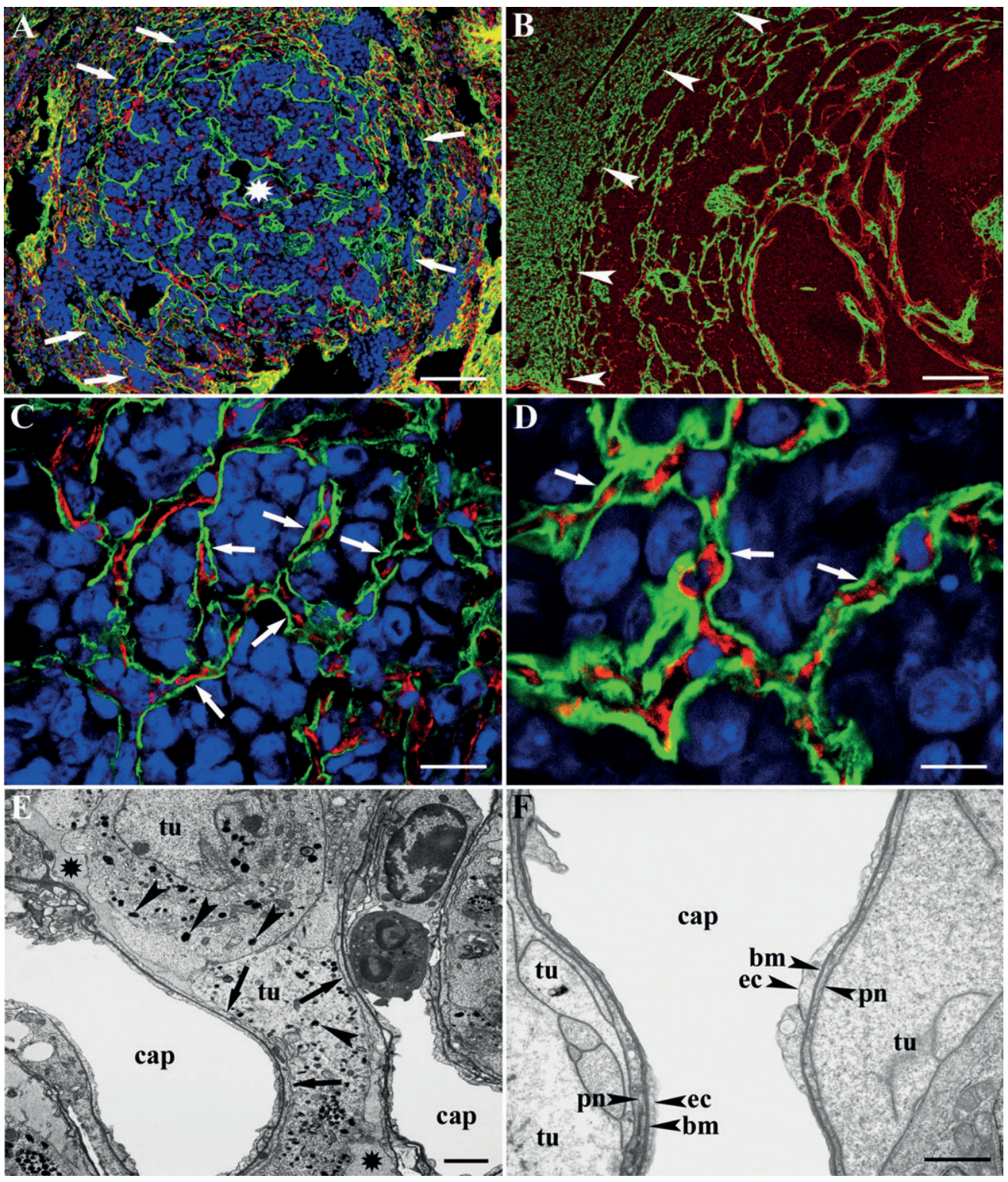

Figure 1. Preservation of alveolar architecture in the peripheral regions of lung metastases that is indicative of vessel co-option. (A) Low-power micrograph of a MAT-B-III lung metastasis from a SCID mouse. The section is stained for the pneumocyte marker podoplanin (green), the EC marker CD31 (red), and TOTO-3 (blue), which highlights the tumour mass. The alveolar air spaces are filled with tumour cells at the tumour periphery (arrows). An asterisk marks the centre of the metastasis. Scale bar $=200 \mu \mathrm{m}$. (B) Micrograph of a C38 lung metastasis stained for CD31 (green) and laminin (red) from a C57BL/6 mouse. Normal lung parenchyma is present on the left (arrowheads mark the border of the metastasis). Tumour cells occupy the alveolar air spaces and expand them. Accordingly, the closer the alveolus is to the tumour centre, the larger the lumen becomes. Scale bar $=200 \mu \mathrm{m}$. (C) Periphery of a MAT-B-III metastasis in a mouse lung stained for podoplanin (green), CD31 (red), and with TOTO-3 (blue). Tumour cells fill the alveoli. Pneumocytes line the surface of the alveoli in a regular manner (arrows). Scale bar $=20 \mu \mathrm{m}$. (D) High-power confocal micrograph of the periphery of an HT1080 lung metastasis formed in a SCID mouse stained for podoplanin (green), CD31 (red), and with TOTO-3 (blue). Note that intact alveolar walls with regular layering (pneumocyte-capillary-pneumocyte) are present (arrows). Scale bar $=10 \mu \mathrm{m}$. (E) Low-power electron micrograph captured at the periphery of a B16 lung metastasis from a C57BL/6 mouse. Tumour cells (tu), which are recognizable due to the presence of melanocytic granules (arrowheads), are seen filling the alveolar air space, whilst the adjacent capillary lumens (cap) are free of tumour cells. Two white blood cells are visible in the lumen of the capillary on the right. The plasma membrane of a tumour cell makes close contact with the pneumocyte plasma membrane over a large surface area (arrows). Asterisks mark areas where the alveolar lumen is filled with protein-containing liquid. Scale bar $=2 \mu \mathrm{m}$. (F). High-power electron micrograph at the periphery of an HT1080 lung metastasis from a SCID mouse showing a capillary (cap) surrounded by tumour cells (tu). On both sides of the capillary, a normal blood-gas barrier is present consisting of a pneumocyte layer (pn), a basement membrane (bm), and an endothelial layer (ec). Scale bar $=1 \mu \mathrm{m}$.

are composed of blood vessels and a sheathing layer of pneumocytes on either side, was strikingly preserved at the periphery of the tumour mass (Figures $1 \mathrm{C}$ and 1D). Alveolar walls present within the tumour were also examined at the electron microscopy level. We found that the blood-gas barrier of the normal lung, which consists of an endothelial cell (EC) layer, a basement membrane (BM), and an epithelial layer, was preserved at the peripheral tumour areas (Figures $1 \mathrm{E}$ and $1 \mathrm{~F}$ ). The lumens of the incorporated capillaries were free of 
tumour cells (Figures $1 \mathrm{E}$ and $1 \mathrm{~F}$ ). These data indicate that the tumour cells gained access to a vascular supply by co-opting the normal alveolar capillaries.

\section{Tumour cells strip the alveolar epithelium from co-opted vessels}

In all the models examined but one (the C38 colorectal cancer model, described later), 'naked' microvessels surrounded by tumour cells (ie blood capillaries not sheathed by a layer of pneumocytes) could be detected $100-200 \mu \mathrm{m}$ inwards from the invasive edge of the metastases (Supplementary Figure 1A). The appearance of these pneumocyte-free blood capillaries occurred due to a unique process instigated by the tumour cells themselves. Tumour cells infiltrated into the alveolar walls and invaded in between the capillaries and the alveolar epithelium (Supplementary Figure 1B). This resulted in detachment of the pneumocytes from the underlying capillaries (Supplementary Figures $1 \mathrm{C}$ and 1E). We used electron microscopy to examine this process at higher resolution and observed tumour cells in the process of separating pneumocytes from ECs. For example, a B16 melanoma cell could be seen extending a protrusive structure that physically drives a wedge between the pneumocyte and the adjacent EC (Figures 2A and 2B). During this process, the BM of the blood-gas barrier was separated into an EC-associated BM layer and an epithelial-associated BM layer (Figures 2A and 2B). After splitting occurred, the tumour cells attached firmly to both the EC-associated BM layer and the epithelial-associated BM layer (Figures 2A and 2B).

The separation of the pneumocyte layer from the endothelial layer was further examined by staining for the $\mathrm{BM}$ component, laminin. In Figure 2C, a capillary (blue) can be seen (small arrow) which still shares its laminin-positive BM (red) with the adjacent pneumocyte layer (arrowhead). However, complete detachment of the epithelium from this capillary is observed at the other end of the vessel. In Figures $2 \mathrm{C}$ and $2 \mathrm{D}$ and also in Supplementary Figure 3A, further capillaries can be observed (large arrows) from which the adjacent pneumocyte layer has been completely detached. It should be noted that whilst both the naked vessel and the adjacent detached pneumocyte layer are associated with a laminin-positive BM, the BM associated with the naked vessel is thicker than the pneumocyte-associated $\mathrm{BM}$ and this can be seen clearly in Figures 2C and $2 \mathrm{D}$.

We also examined the presence of pneumocytes in the metastases as a function of the lesion size. Whilst the central area of smaller metastases contained pneumocytes (Figure 2E), the central area of larger metastases was generally devoid of alveolar epithelial cells (Figure 2F). It is apparent that after they were detached from the microvessels, these alveolar epithelial cells underwent fragmentation and disappeared (Figure 2F). In contrast, the denuded and incorporated microvessels survived. These vessels appear to be functional as extensive BrdU incorporation was observed in tumour cells surrounding these microvessels even in central tumour areas (Figure 2G).

According to the angiogenic switch theory, tumours must activate new vessel growth to grow beyond $1-2 \mathrm{~mm}$ in size [18]. Although the majority of the metastases that we studied in the mouse did not reach this critical size, MAT-B-III tumours growing in rat lung did exceed this size limit (mean diameter $3.36 \pm 2.23 \mathrm{~mm}$; range $0.3-14.8 \mathrm{~mm}$; median $2.8 \mathrm{~mm}$ ). To determine whether these tumours activated the growth of new vessels, we examined these tumours for EC proliferation. We compared the proliferation rate of ECs in the peritumoural region (a band of normal lung tissue $100 \mu \mathrm{m}$ wide that was directly adjacent to the metastases) versus intratumoural regions of MAT-B-III rat lung metastases. Peritumoural ECs showed a negligible proliferation rate $(1.73 \pm 0.8 \%$ of ECs), but the proliferation of ECs was moderately increased intratumourally $(12.8 \pm 3.2 \%)$. The difference in proliferation was statistically significant $(p<0.05)$. However, we detected a slight increase in microvessel perimeters towards the tumour core compared with the periphery, suggesting that EC proliferation may result in vessel dilatation (data not shown).

Increased desmoplastic reaction towards the centre of C38 metastases results in the transformation of alveolar walls into intratumoural tissue columns

As mentioned above, the C38 model of lung metastasis demonstrated a different behaviour to the other models. C38 tumour cells did not reinvade the alveolar walls and thus they could not detach the pneumocytes from the alveolar capillaries; instead, these tumours incorporated the alveolar walls 'as a whole' and induced a desmoplastic response in them. As a result, in C38 metastases, alveolar walls were continuously transformed into intratumoural tissue columns (centrally located capillaries embedded in connective tissue collagen and $\alpha$ SMA-expressing activated fibroblasts surrounded by a BM) (Figures 3A and 4B). These structures developed gradually in the direction from the periphery towards the tumour centre (Figure 3A-3D). First, in the walls of alveoli (of which the lumens were filled with tumour mass), $\alpha$ SMA-expressing activated fibroblasts appeared (of note, no such cells - except within the arterioles and airways - were present in the peritumoural normal lung parenchyma). The number of activated fibroblasts increased towards the tumour centre (Figures 3A-3D). Consequently, the amount of deposited connective tissue collagen and, in turn, the space between the capillaries and the epithelium (Figures 4A and 4B) also increased. Importantly, C38 cells did not invade this space (ie the tissue columns); they remained in the alveolar lumens (Figure 4C). During the development of connective tissue columns, the epithelium facing the tumour 

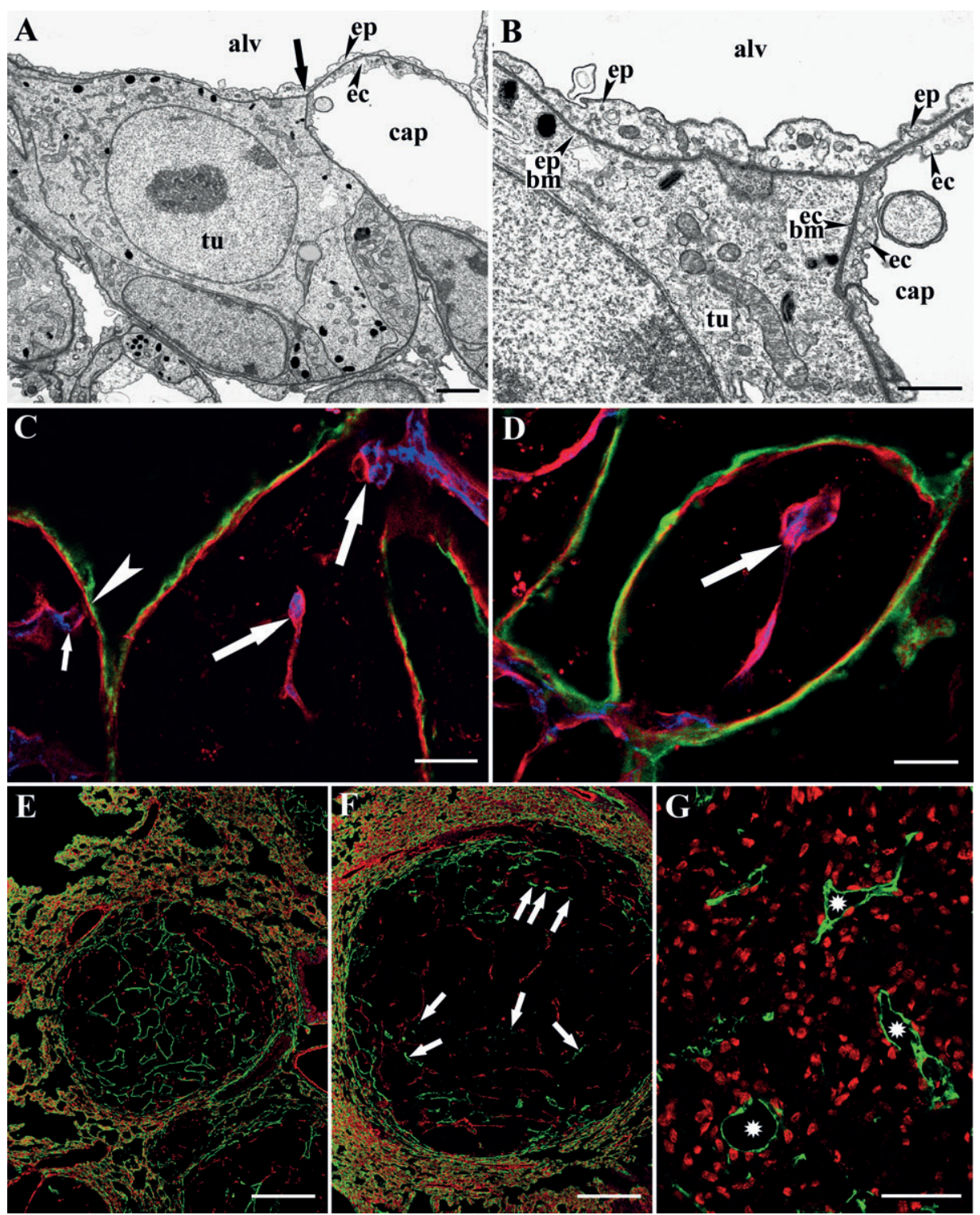

Figure 2. Tumour cells split the basement membrane of the co-opted blood-gas barrier. $(A, B)$ Electron micrographs of a B16 lung metastasis. In $A$, a tumour cell ( $\mathrm{tu}$ ) is shown in the process of detaching the epithelium from the surface of a capillary. A wedge-shaped cellular process of the tumour cell is advancing between the epithelial (ep) and EC (ec) layers (arrow). In B, the area where the arrow is pointing in A is shown at high power. Note how the wedge-shaped process splits the alveolar BM into an EC-associated BM and an epithelial-associated BM. alv = alveolar space; $b m=$ basement membrane; $\mathrm{ep}=$ epithelium; $\mathrm{ec}=$ endothelial cell; $\mathrm{cap}=$ capillary lumen; $\mathrm{tu}=$ tumour cell. Scale bars: (A) $2 \mu \mathrm{m}$; (B) $1 \mu \mathrm{m}$. (C, D) Images of an HT1080 lung metastasis stained formicrovessels (CD31, blue), BM (laminin, red), and pneumocytes (podoplanin, green). The black areas are filled with tumour cells (the presence of tumour cells in these black areas within an HT1080 metastasis is demonstrated in Supplementary Figures 3A-3C). In C, on the left-hand side, a microvessel (small arrow) can be seen that is still associated with the epithelium (arrowhead). In C, microvessels marked by large arrows are completely separated from the epithelium and are surrounded by the tumour mass. Note that the detached alveolar epithelium (green) lies on its own BM (red). (D) A completely denuded microvessel (arrow) within an HT1080 metastasis is shown. CD31-positive ECs (blue) are surrounded by a thick BM (laminin, red). Note that in both $C$ and $D$, the BM layer (laminin, red) that supports the podoplanin-positive epithelium (green) is thinner than the BM layer (laminin, red) that supports the microvessels (CD31, blue). Scale bars $=20 \mu \mathrm{m}$. A similar area of an HT1080 metastasis stained for vimentin, laminin, and CD31 is shown in Supplementary Figure 3A. (E, F) Image of a small (E) and a large (F) B16 metastasis stained for podoplanin (green) and CD31 (red). In addition to the presence of blood capillaries, the smaller metastasis (E) contains centrally located sheets of alveolar epithelium. In contrast, pneumocytes are present only at the tumour periphery in the larger metastasis (F). Arrows indicate fragmented epithelium. The presence of numerous centrally located microvessels (red) raises the possibility of neo-angiogenesis. Scale bars $=200 \mu \mathrm{m}$. HEtE staining of a B16 metastasis is shown in Supplementary Figure 3D. (G) BrdU labelling (red) of an HT1080 lung metastasis. Microvessels are highlighted by CD31 (green). BrdU-positive tumour cells are preferably localized around the microvessels (asterisks). Scale bar $=50 \mu \mathrm{m}$.

became gradually fragmented (Figures 4C and 4D). It is unclear whether the BM surrounding the intratumoural connective tissue columns was the original epithelial $\mathrm{BM}$ or whether it was newly synthesized by the tumour.

\section{Presence of stromal fibroblasts and connective} tissue within the other lung metastasis models

Importantly, the distribution of $\alpha$ SMA-positive myofibroblasts and connective tissue collagen was different 

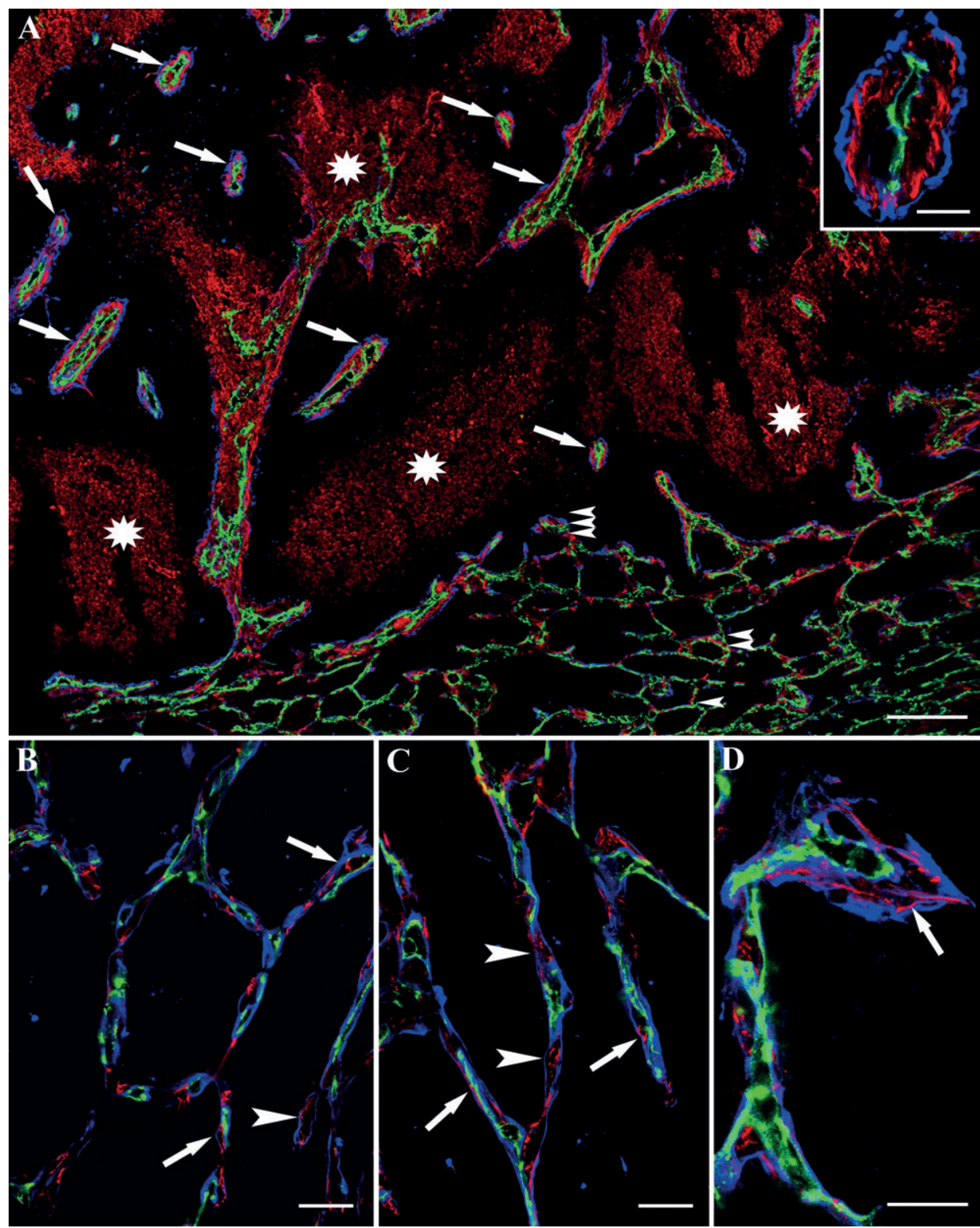

Figure 3. Increased desmoplastic reaction towards the centre of C38 metastases results in the transformation of alveolar walls into intratumoural tissue columns (I). (A) C38 metastasis stained for CD31 to mark vessels (green), $\alpha$ SMA to mark activated myofibroblasts (red), and laminin to mark the BM (blue). Black areas represent viable tumour tissue throughout the picture. Asterisks mark areas of necrotic tissue that also appear red due to non-specific binding of the anti-mouse secondary antibody. The growing tumour is continuously incorporating and transforming the alveolar walls (in the lower part of the picture) into tissue columns (arrows) with central microvessel(s). Within these tissue columns, vascular remodelling and sprouting angiogenesis might also be present [43]. Note that no necrosis can be seen at the tumour periphery where alveoli are still visible. In the alveolar walls shown in the lower right area of the picture, $\alpha S M A-p o s i t i v e$ cells cannot be detected. Such cells appear gradually towards the tumour centre. The inset shows a column at high power with a single central capillary. Scale bars: (A) $100 \mu \mathrm{m}$; (A, inset) $20 \mu \mathrm{m}$. (B-D) High-power micrographs of the regions marked by a single arrowhead (B), double arrowheads (C), and triple arrowheads (D) in A. Alveolar lumens are filled with tumour cells (black areas represent the tumour mass). The number of $\alpha$ SMA-positive cells (red) within the alveolar walls increases towards the centre of the tumour through B-D. These cells are present within the alveolar walls between the epithelial BM (laminin, blue) and the capillary (CD31, green) (arrows) or between the epithelial BMs (laminin, blue) (arrowheads). In D, note the increased number of $\alpha S M A$-expressing fibroblasts (red) between the epithelial and capillary BM layers (arrow). Scale bar: (B-D) $20 \mu \mathrm{m}$. The presence of tumour cells in black areas within a C38 metastasis is shown in Supplementary Figures 3E-3G.

in HT1080, HT25, B16, C26, and MAT-B-III compared with the C38 model. In these former models, an extensive desmoplastic reaction was not observed. $\alpha$ SMA-positive myofibroblasts did become incorporated into the metastases in association with the alveolar capillaries; however, they did not participate in the development of intratumoural tissue columns. The $\alpha$ SMA-positive myofibroblasts were in close contact with the BM of the microvessels (Supplementary Figures 2A and 2B). A minority of these cells, however, were denuded from the microvessels along with the alveolar epithelial BM (Supplementary Figure 2A). The number of $\alpha$ SMA-positive cells did not increase towards the centre of the tumour in the B16 melanoma 

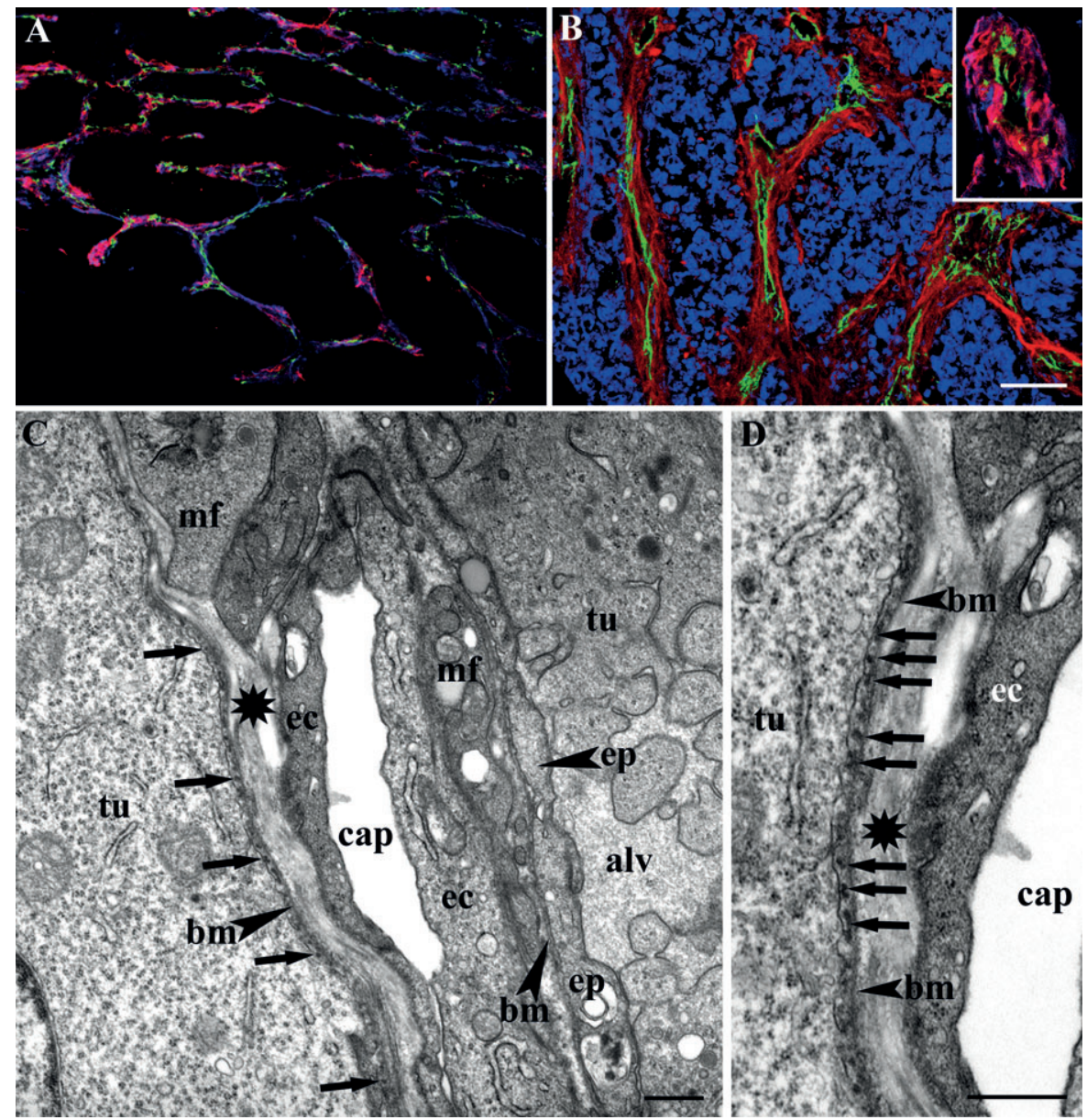

Figure 4. Increased desmoplastic reaction towards the centre of C38 metastases results in the transformation of alveolar walls into intratumoural tissue columns (II). (A) Periphery of a C38 metastasis. The section is stained for CD31 (green), collagen I (red), and $\alpha$ SMA (blue). The alveoli are filled with tumour cells (black areas). $\alpha$ SMA-positive activated fibroblasts (blue) are present in the walls of the alveoli. Collagen I (red) is deposited in an increasing amount towards the centre of the tumour (on the left). The distribution of the $\alpha$ SMA-expressing cells and the connective tissue collagen shows that the appearance of the activated fibroblasts at the tumour periphery precedes the deposition of collagen. (B) Centre of a C38 metastasis. The section is stained for CD31 (green), collagen I (red), and TOTO-3 (blue), which highlights tumour cells. Massive and contiguous collagen I-positive connective tissue septa and columns are present within the tumour. These structures might provide a suitable environment for sprouting angiogenesis [43]. The inset shows the co-localization of aSMA-expressing activated fibroblasts (blue), collagen I-positive connective tissue (red), and CD31-positive ECs (green). Scale bar: (A, B) $50 \mu \mathrm{m}$. (C) Electron micrograph of an alveolar wall surrounded by C38 cells. The alveolar space (alv) on the right is partially filled by tumour cells (tu). Epithelial cells (ep) cover the alveolar wall at this location. Fragmented epithelium (arrows) is present on the alveolar wall on the left where the tumour cell (tu) is located close to the epithelial BM (bm). In the alveolar wall beside the myofibroblast (mf), connective tissue collagen (asterisk) is also present. cap = capillary. Scale bar $=1 \mu \mathrm{m}$. (D) The alveolar wall shown in C. Fragments of the epithelium (arrows) are visible between the C38 cell (tu) and the BM (bm). Scale bar (C, D): $1 \mu \mathrm{m}$.

and HT1080 fibrosarcoma. In contrast, the number of activated fibroblasts increased from peritumoural lung tissue towards the tumour centre in MAT-B-III, C26, and especially in HT25 colon carcinoma metastases (data not shown).

In all tumour types, the $\alpha$ SMA-positive activated fibroblasts co-distributed with connective tissue collagen (Supplementary Figure 2C and inset of Figure 4B). A small amount of connective tissue collagen was always deposited around the incorporated microvessels in the B16 melanoma and HT1080 fibrosarcoma (Supplementary Figures 2C and 2D). Scattered collagen I positivity could also be observed in the MAT-B-III mammary carcinoma and C26 colon carcinoma metastases (Supplementary Figures 2E and 2F). The HT25 colon carcinoma metastases contained larger amounts of collagenous connective tissue (Supplementary Figure 2G).

\section{Lung metastases develop an arterial blood supply}

Because the lung has a dual circulation system, we also analysed the origin of the blood supply of lung metastases. The anastomosis system between the bronchial and the pulmonary circulation is quite similar to that observed previously by us in the mouse liver [28]. Accordingly, we applied the same corrosion casting method [28]. In accordance with other investigators [26,27], we could not identify bronchial arteries downstream from the main bronchi in mice (data not shown). Therefore, we used the MAT-B-III rat mammary carcinoma system. In the rat lung, only the main arterial 


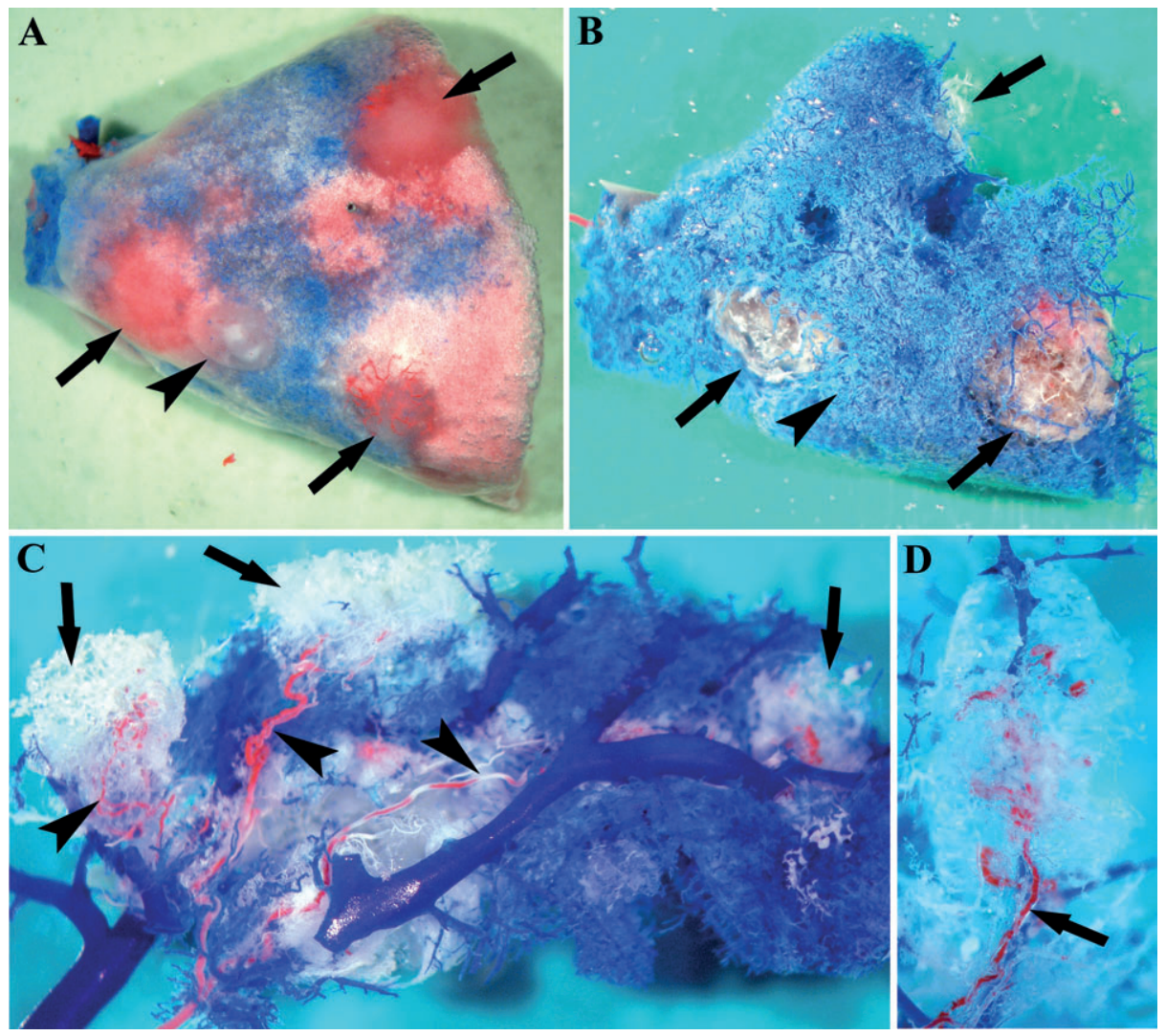

Figure 5. Lung metastases develop an arterial blood supply. (A-D) Corrosion casting of MAT-B-III mammary carcinoma metastases within rat lungs. $A$ and $B$ show the right lower pulmonary lobe with metastases before $(A)$ and after $(B)$ corrosion. The lung was filled first through the pulmonary artery (blue), followed by filling through the bronchial arterial system (red). The lobe contains three metastases with and one without a bronchial arterial blood supply (arrows and arrowhead, respectively). Because the metastasis in the upper part of the picture was incompletely filled, only part of it is visible after corrosion. (C) The left pulmonary lobe with metastases after corrosion. For better visibility, branches of the pulmonary system were removed. Three metastases with a bronchial arterial blood supply (arrows) are visible. Each has its own branch of the bronchial artery (arrowheads). (D) Metastasis with an arterial blood supply. Note the central position of the feeding artery (arrow).

branches could be filled and thus it was not possible to decide whether segmental bronchi have an arterial blood supply. Investigating the origin of the blood supply in metastases with different sizes, we found that $95 \%$ of the metastases with a diameter greater than $5 \mathrm{~mm}$ had an arterial blood supply and that $97 \%$ of the metastases with a diameter less than $3 \mathrm{~mm}$ had a pulmonary blood supply (Figures 5A and 5B). Sixty-five per cent of the arterioles supplying the tumour cells were centrally positioned in the metastases (Figures 5C and 5D). We also compared the proliferation rate of the metastatic cells: the tumour cell labelling index of metastases with a bronchial blood supply was significantly higher than that of metastases with a pulmonary blood supply $(43.5 \pm 6.1 \%$ versus $35.3 \pm 2.5 \%$, respectively; mean $\pm \mathrm{SD} ; p<0.05$ ).

\section{Discussion}

\section{Experimental lung metastases can vascularize using vessel co-option}

Angiogenesis (the development of new blood vessels from pre-existing ones) has long been regarded as crucial for tumour growth $[16-18,29]$. However, it is today obvious that tumours can acquire their blood supply via alternative (non-sprouting) mechanisms [10], including glomeruloid microvessel growth [30], vasculogenic mimicry [31], intussusceptive microvascular growth [32], postnatal vasculogenesis [33], and vessel co-option [34]. In particular, there are also reports on the presence and clinical significance of alternative vascularization mechanisms in the lungs. Tanaka et al demonstrated that glomeruloid vessel formation was associated with an aggressive angiogenic phenotype and significantly reduced survival in NSCLC patients [35]. Wu et al identified vasculogenic mimicry in 35\% of NSCLC patients and, furthermore, provided evidence for an association of vasculogenic mimicry with clinical outcome in this malignancy [36]. Although intussusceptive microvascular growth was first observed in the growing postnatal lung [37,38] and then also during postpneumonectomy lung growth [39], there are no reports on this mechanism in pulmonary malignancies. Altogether, however, vessel co-option is the most frequently reported vascularization mechanism in human lung tumour pathology studies [19-24].

In the present study, we analysed the vascularization process in six different experimental models of 

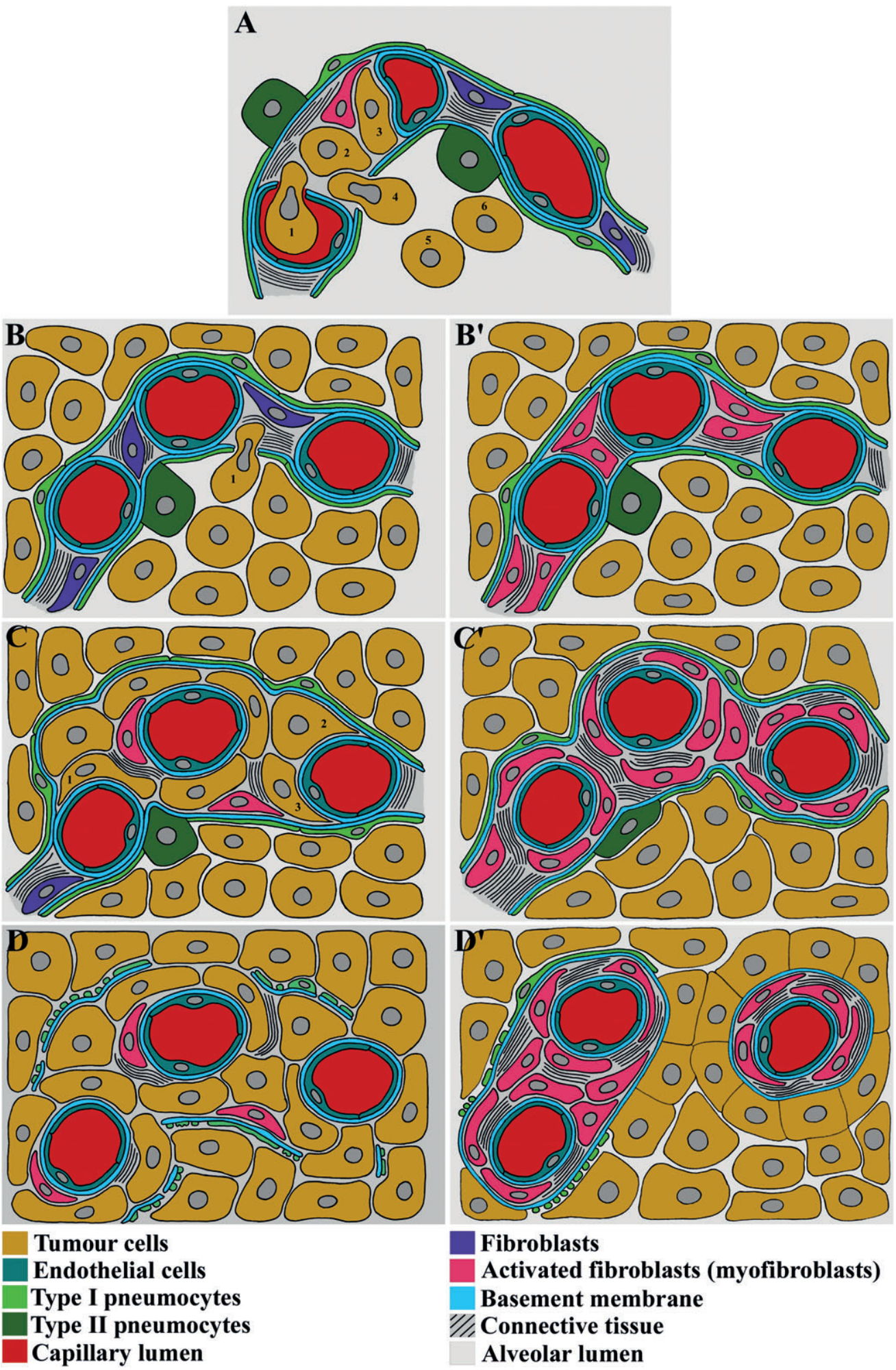

Fibroblasts

Activated fibroblasts (myofibroblasts)

Basement membrane

//, Connective tissue

Alveolar lumen

Figure 6. Legend on the next page.

lung metastasis. Strikingly, in all models utilized, the tumours became vascularized via the process of vessel co-option. All of the investigated cell lines incorporated the pre-existing host tissue capillaries within the alveolar walls, without morphological signs of active peritumoural EC sprouting. This latter finding is further supported by our observation that peritumoural ECs exhibited a low proliferation rate. The absence of peritumoural EC sprouting is most probably due to the high pulmonary tissue capillary densities and, moreover, to the lack of a suitable amount of connective tissue matrix in the alveolar walls $[40,41]$. It is possible, however, that intratumoural EC sprouting may occur at later stages of tumour development (provided that suitable connective tissue is available within the more central areas of the metastases). This assumption is supported 
by our finding of significantly elevated intratumoural EC proliferation (versus the peritumoural lung) in the MAT-B-III rat mammary carcinoma metastases. Nevertheless, in addition to providing a source of new capillary production, the elevated EC proliferation may merely lead to vessel dilatation. In support of this, we previously showed that something similar occurs in a murine skin melanoma model [34].

\section{Tumour cells of experimental lung metastases can strip the epithelium from co-opted blood vessels}

A striking finding from this study is the observation that tumour cells can strip the epithelium from the co-opted alveolar walls. This was observed in the B16, HT1080, HT25, C26, and MAT-B-III models. In these tumour types, malignant cells were observed to invade into the alveolar walls and migrate between the endothelial and epithelial layers (Figure 6). After splitting occurred, the tumour cells attached firmly to both the endothelial-associated BM layer and the epithelial-associated BM layer. The stripped epithelial cells were highly compressed and then underwent a process of fragmentation and disappeared from the metastasis, whereas the denuded capillaries remained functional and were able to ensure the blood supply of the metastases. The split of the alveolar BM by cancer cells is an unusual phenomenon which we do not believe has been described before. The exact molecular mechanism through which this process occurs is currently unclear, but presumably involves selective adhesion between the tumour cells and the BM of the blood-gas barrier, as well as the expression of proteases that may be acting to digest the BM in a highly selective fashion to split the BM in two.

In the invasively growing cell lines, the majority of alveolar wall fibroblasts remained associated with the capillaries. However, we do not believe that they were functioning as pericytes as they did not have BM coverage. Instead, we propose that they were only responsible for collagen deposition within the metastases. This finding might be important because pericytefree capillaries may be more vulnerable against VEGF withdrawal than their normal counterparts [42].

\section{Generation of desmoplastic tissue columns in the C38 lung metastasis model}

Tumour cells of the C38 lung metastasis model also incorporated the alveolar walls. However, instead of re-invading the alveolar interstitium, these cells elicited a desmoplastic response within the incorporated alveolar walls resulting in the transformation of alveolar walls into microvessel-containing connective tissue columns. The activation and increased number of interstitial fibroblasts (and the accompanying collagenous matrix deposition) at the tumour periphery led to the gradual widening of the space between the two BM layers (endothelial and epithelial/tumoural) towards the tumour centre. This process assumes that the BM of the blood-gas barrier in this tumour type is dissected into two layers by the proliferating myofibroblasts. In parallel, the alveolar epithelium became fragmented and disappeared and thus C38 cells could attach directly to the BM left over by the epithelial cells. It is also possible that $\mathrm{C} 38$ cells synthesized their own BM material onto the original BM. Although we do not have specific antibodies against these BMs, the latter scenario is indeed more probable as C38 cells in other locations always synthesize their own BM onto the surface of the incorporated connective tissue [43]. In either case, C38 cells use BM material as a substrate for their growth.

All in all, the transformation process of the alveolar walls in the C38 model culminated in the appearance of connective tissue columns with a single

Figure 6. Schematic representation of the vascularization process in lung metastases. (A) The common steps of the vascularization process among the different lung metastasis models utilized in this study are shown. A tumour cell arrested in a lung capillary extravasates into the alveolar interstitium (tumour cell \#1). After forming small colonies in the interstitial connective tissue (tumour cells \#2 and \#3), tumour cells enter the alveolar lumen (tumour cell \#4) and proliferate further there (tumour cells \#5 and \#6) (also see refs 50-54). Note that where connective tissue material is present in the alveolar walls, the BM of the blood-gas barrier is separated into an endothelial BM layer and an epithelial BM layer also in the tumour cell-free part of the alveolar wall. Of note, since the tumour mass advances from one alveolar lumen to another, the events depicted in B-D take place in alveoli distant from the first entrapped alveolus. (B-D) The invasively growing tumour cell lines (B16, HT1080, HT25, C26, and MAT-B-III) acquire their blood vasculature by stripping the alveolar capillaries from the epithelium. (B) Tumour cells proliferate intensively in the occupied alveolar lumens and (re-)invade the alveolar wall connective tissue (tumour cell \#1). (C) Tumour cells in the alveolar walls start to detach the epithelium (together with the epithelial BM) from the capillaries (tumour cells \# 1-3). This process culminates in the appearance of completely denuded capillaries (middle capillary) surrounded by tumour cells. The two other capillaries are still partly covered by the epithelium. (D) Capillaries are denuded and completely surrounded by tumour cells. Remnants of the fragmented epithelium are scattered within the tumour mass. (B' - D') Vascularization of C38 colon cancer metastases is accompanied by a desmoplastic response in the co-opted alveolar walls. In $B^{\prime}-D^{\prime}$, the alveolar spaces depicted are distant from the initial extravasation place; $B^{\prime}-D^{\prime}$ represent areas in the direction from the periphery towards the centre of the metastases. ( $\left.B^{\prime}\right)$ C38 cells proliferate in the alveolar lumens at the periphery of the metastases. In the walls of the occupied alveolar spaces, fibroblasts become activated ( $\alpha \mathrm{SMA}$-expressing myofibroblasts) but deposition of collagen is not yet started. ( $\mathrm{C}^{\prime}$ ) Towards the centre of the metastases, a desmoplastic response is elicited in the alveolar walls: the number of myofibroblasts increases and collagenous connective tissue matrix is deposited. (D') As the incorporated alveolar walls get closer to the centre of the metastases, they undergo further 'processing'. The tumour mass divides the desmoplastic alveolar wall into pieces, leading to the appearance of separate intratumoural connective tissue columns. Pneumocytes are fragmented (this process can be seen on the surface of the connective tissue column on the left side of the picture) and the tumour cells attach to the residual BM of the pneumocytes or synthesize their own BM onto the surface of the extracellular matrix of the tissue columns. Throughout the drawings, parallel black lines represent collagen bundles in the connective tissue. 
central microvessel. Although the final steps of tissue column development are unclear, one possibility is that the originally incorporated alveolar walls with more microvessels are further 'processed' by the tumour, which may split the tissue columns into more parts until the single microvessel stage is reached. However, this assumes continuous myofibroblast proliferation and connective tissue deposition. It is also important to mention that sprouting of connective tissue column capillaries following collagenous matrix deposition is also possible, as demonstrated previously by our group in C38 liver metastases [43].

\section{Arterialization of lung metastases}

In a recent study, we found that mouse liver metastases inevitably become arterialized [28]. The results of the present study suggest that pulmonary metastases develop an arterial blood supply as well. The growing tumour incorporates a high-pressure bronchial vessel which, akin to hepatic arterioles, frequently acquires a central intratumoural position. Thus, the process is similar to that of the hepatic metastases, where it is purely mechanical in nature, governed by the pressure relationships in the liver vasculature. The key step in the process of liver metastasis arterialization is the fusion of the sinusoids at the tumour-liver parenchyma interface [28]. Although we could not confirm a similar phenomenon (ie capillary fusion) in the present study, chances are that where the disruption of sphincters between the sinusoids and terminal arterioles resulted in an increase in the intratumoural blood pressure, metastatic tumour cells were also likely to damage the Sperr arteries (short arterial segments that can connect the bronchial and pulmonary circulation) $[44,45]$, which in turn may let the high-pressure bronchial arterial bloodstream enter the vascular system of the metastases.

MAT-B-III cells of arterialized metastases had a significantly higher proliferation rate than those of tumours supplied with pulmonary blood. Although the mechanisms that result in the relatively lower tumour cell proliferation rate in the metastases with a pulmonary blood supply are not completely understood, a possible explanation can be deducted from several studies demonstrating that hypoxia can slow down or even completely inhibit tumour cell proliferation both in vitro and in vivo [46-48]. It is also important to note that there should be other factors influencing the proliferation rate of metastatic cells besides the origin of the blood supply. For example, the proliferation rate of 3LL-HH and C38 cells of liver metastases with a baseline labelling index above $50 \%$ was not accelerated further by arterial blood [28]. In contrast, hepatic A2058 melanoma metastases [28] and pulmonary MAT-B-III breast cancer metastases had a relatively lower labelling index which could be further increased by a switch from portal to arterial and from a pulmonary to a bronchial blood supply, respectively.
Consequences for the clinical management of lung metastases

Metastasis to the lungs is a key event in malignant disease outcome and the treatment of pulmonary metastases remains a major clinical challenge [5-9]. Our study describes the mechanism of vascularization of pulmonary metastases and provides the first experimental evidence that similar to primary human non-small cell lung cancers $[19,21]$ and lung metastases of human breast, colorectal, and renal cancer [20,22,24], these tumours can co-opt the host alveolar capillaries during their growth. Our study, however, does not only provide evidence that the characteristic 'alveolar growth pattern' described in human pulmonary metastases $[20,24]$ can be mimicked in appropriate in vivo models but also raises serious questions regarding the utility of using anti-angiogenic agents for the treatment of lung metastases. The conventional generation of anti-angiogenic agents, such as bevacizumab and sunitinib, is designed to target the process of sprouting angiogenesis and it is unclear whether these therapies would be successful against lung metastases that vascularize via vessel co-option.

Novel invasive strategies including transcatheter chemoembolization therapies have been developed recently to improve the clinical management of pulmonary metastases $[9,49]$. Importantly, it is unclear if primary and secondary lung tumours have a pulmonary and/or a bronchial blood supply. Accordingly, there is no established protocol for chemoembolization in these settings (ie it remains to be investigated if these tumours should be catheterized/perfused via the pulmonary or the bronchial system) $[9,49]$. Our results show that tumour size influences the extent of the pulmonary versus arterial blood supply that a lung metastasis receives, which may be an important consideration for the future application of transcatheter chemoembolization therapies in secondary lung tumours.

\section{Acknowledgments}

We were supported by KTIA AIK 12-1-2013-0041 (EB, JTo, VL, BD, SP); TÁMOP 4.2.4.A/1-11-1-2012-0001 (KD); OTKA K109626, OTKA K108465 (BD), and OTKA K84173 (JTo); the National Development Agency under the Research and Technology Innovation Fund (EUREKA_HU_12-1-2012-0057; BD); and the Vienna Fund for Innovative Interdisciplinary Cancer Research (BD, VL). AR, SF, EW, and VB acknowledge support from Breakthrough Breast Cancer and the NIHR Royal Marsden/Institute of Cancer Research Biomedical Research Centre.

\section{Author contributions statement}

PBV, ARR, BD, JTo, JTi, PN, and SP conceived the study and/or contributed to the interpretation of the data. 
$\mathrm{BD}, \mathrm{SP}$, and ARR wrote the manuscript. VL, VS, NE, and $\mathrm{KD}$ were responsible for generating the in vivo lung metastases and assessed tumour and endothelial cell proliferation rates. EB, VLB, EW, and SF performed the corrosion casting and the confocal and electron microscopic analysis. All authors read and approved the manuscript.

\section{References}

1. Krishnan K, Khanna C, Helman LJ. The molecular biology of pulmonary metastasis. Thorac Surg Clin 2006; 16: 115-124.

2. Snyder BJ, Pugatch RD. Imaging characteristics of metastatic disease to the chest. Chest Surg Clin N Am 1998; 8: 29-48.

3. Davis SD. CT evaluation for pulmonary metastases in patients with extrathoracic malignancy. Radiology 1991; 180: 1-12.

4. Crow J, Slavin G, Kreel L. Pulmonary metastasis: a pathologic and radiologic study. Cancer 1981; 47: 2595-2602.

5. Schweiger T, Lang G, Klepetko W, et al. Prognostic factors in pulmonary metastasectomy: spotlight on molecular and radiological markers. Eur J Cardiothorac Surg 2014; 45: 408-416.

6. Pastorino U, Buyse M, Friedel G, et al. Long-term results of lung metastasectomy: prognostic analyses based on 5206 cases. The International Registry of Lung Metastases. J Thorac Cardiovasc Surg 1997; 113: 37-49.

7. Bar J, Herbst RS, Onn A. Targeted drug delivery strategies to treat lung metastasis. Expert Opin Drug Deliv 2009; 6: 1003-1016.

8. Zheng Y, Fernando HC. Surgical and nonresectional therapies for pulmonary metastasis. Surg Clin North Am 2010; 90: 1041-1051.

9. Vogl TJ, Shafinaderi M, Zangos S, et al. Regional chemotherapy of the lung: transpulmonary chemoembolization in malignant lung tumors. Semin Intervent Radiol 2013; 30: 176-184.

10. Dome B, Hendrix MJ, Paku S, et al. Alternative vascularization mechanisms in cancer: pathology and therapeutic implications. $A m$ J Pathol 2007; 170: 1-15.

11. Minchinton AI, Tannock IF. Drug penetration in solid tumours. Nature Rev Cancer 2006; 6: 583-592.

12. Moreno Garcia V, Basu B, Molife LR, et al. Combining antiangiogenics to overcome resistance: rationale and clinical experience. Clin Cancer Res 2012; 18: 3750-3761.

13. Mountzios G, Pentheroudakis G, Carmeliet P. Bevacizumab and micrometastases: revisiting the preclinical and clinical rollercoaster. Pharmacol Ther 2014; 141: 117-124.

14. Ebos JM, Kerbel RS. Antiangiogenic therapy: impact on invasion, disease progression, and metastasis. Nature Rev Clin Oncol 2011; 8: 210-221.

15. Vasudev NS, Reynolds AR. Anti-angiogenic therapy for cancer: current progress, unresolved questions and future directions. Angiogenesis 2014; 17: 471-494.

16. Folkman J. Tumor angiogenesis: therapeutic implications. $N$ Engl $J$ Med 1971; 285: 1182-1186.

17. Folkman J. What is the evidence that tumors are angiogenesis dependent? J Natl Cancer Inst 1990; 82: 4-6.

18. Folkman J, Merler E, Abernathy C, et al. Isolation of a tumor factor responsible for angiogenesis. J Exp Med 1971; 133: 275-288.

19. Pezzella F, Pastorino U, Tagliabue E, et al. Non-small-cell lung carcinoma tumor growth without morphological evidence of neo-angiogenesis. Am J Pathol 1997; 151: 1417-1423.

20. Pezzella F, Di Bacco A, Andreola S, et al. Angiogenesis in primary lung cancer and lung secondaries. Eur J Cancer 1996; 32A: 2494-2500.

21. Adighibe $\mathrm{O}$, Micklem $\mathrm{K}$, Campo $\mathrm{L}$, et al. Is nonangiogenesis a novel pathway for cancer progression? A study using 3-dimensional tumour reconstructions. Br J Cancer 2006; 94: 1176-1179.
22. Breast Cancer Progression Working Party. Evidence for novel non-angiogenic pathway in breast-cancer metastasis. Lancet 2000; 355: 1787-1788.

23. Yousem SA. Peripheral squamous cell carcinoma of lung: patterns of growth with particular focus on airspace filling. Hum Pathol 2009; 40: $861-867$.

24. Sardari Nia P, Hendriks J, Friedel G, et al. Distinct angiogenic and non-angiogenic growth patterns of lung metastases from renal cell carcinoma. Histopathology 2007; 51: 354-361.

25. Donnem T, Hu J, Ferguson M, et al. Vessel co-option in primary human tumors and metastases: an obstacle to effective anti-angiogenic treatment? Cancer Med 2013; 2: 427-436.

26. Mitzner W, Lee W, Georgakopoulos D, et al. Angiogenesis in the mouse lung. Am J Pathol 2000; 157: 93-101.

27. Verloop MC. On the arteriae bronchiales and their anastomosing with the arteria pulmonalis in some rodents; a micro-anatomical study. Acta Anat (Basel) 1949; 7: 1-32.

28. Dezso K, Bugyik E, Papp V, et al. Development of arterial blood supply in experimental liver metastases. Am J Pathol 2009; 175: 835-843.

29. Hanahan D, Weinberg RA. Hallmarks of cancer: the next generation. Cell 2011; 144: 646-674.

30. Dome B, Timar J, Paku S. A novel concept of glomeruloid body formation in experimental cerebral metastases. J Neuropathol Exp Neurol 2003; 62: 655-661.

31. Maniotis AJ, Folberg R, Hess A, et al. Vascular channel formation by human melanoma cells in vivo and in vitro: vasculogenic mimicry. Am J Pathol 1999; 155: 739-752.

32. Paku S, Dezso K, Bugyik E, et al. A new mechanism for pillar formation during tumor-induced intussusceptive angiogenesis: inverse sprouting. Am J Pathol 2011; 179: 1573-1585.

33. Dome B, Timar J, Ladanyi A, et al. Circulating endothelial cells, bone marrow-derived endothelial progenitor cells and proangiogenic hematopoietic cells in cancer: from biology to therapy. Crit Rev Oncol Hematol 2009; 69: 108-124.

34. Dome B, Paku S, Somlai B, et al. Vascularization of cutaneous melanoma involves vessel co-option and has clinical significance. J Pathol 2002; 197: 355-362.

35. Tanaka F, Oyanagi H, Takenaka K, et al. Glomeruloid microvascular proliferation is superior to intratumoral microvessel density as a prognostic marker in non-small cell lung cancer. Cancer Res 2003; 63: 6791-6794.

36. Wu S, Yu L, Wang D, et al. Aberrant expression of CD133 in non-small cell lung cancer and its relationship to vasculogenic mimicry. BMC Cancer 2012; 12: 535.

37. Burri PH, Tarek MR. A novel mechanism of capillary growth in the rat pulmonary microcirculation. Anat Rec 1990; 228: 35-45.

38. Caduff JH, Fischer LC, Burri PH. Scanning electron microscope study of the developing microvasculature in the postnatal rat lung. Anat Rec 1986; 216: 154-164.

39. Ackermann M, Houdek JP, Gibney BC, et al. Sprouting and intussusceptive angiogenesis in postpneumonectomy lung growth: mechanisms of alveolar neovascularization. Angiogenesis 2014; 17: 541-551.

40. Low FN. Extracellular components of the pulmonary alveolar wall. Arch Intern Med 1971; 127: 847-852.

41. Rosario EJ, Lewis CW, Gureenberg SD, et al. The architecture of the alveolar wall: an ultrastructural study in man. Cardiovasc Res Cent Bull 1973; 12: 13-20.

42. Erber R, Thurnher A, Katsen AD, et al. Combined inhibition of VEGF and PDGF signaling enforces tumor vessel regression by interfering with pericyte-mediated endothelial cell survival mechanisms. FASEB J 2004; 18: 338-340. 
43. Paku S, Kopper L, Nagy P. Development of the vasculature in 'pushing-type' liver metastases of an experimental colorectal cancer. Int J Cancer 2005; 115: 893-902.

44. Hayek Hv. The Human Lung. Hafner Pub Co: New York, 1960.

45. Schraufnagel DE, Pearse DB, Mitzner WA, et al. Three-dimensional structure of the bronchial microcirculation in sheep. Anat Rec 1995; 243: $357-366$.

46. Giaccia AJ. Hypoxic stress proteins: survival of the fittest. Semin Radiat Oncol 1996; 6: 46-58.

47. Siemann DW, Keng PC. Characterization of radiation resistant hypoxic cell subpopulations in KHT sarcomas. (II). Cell sorting. $\mathrm{Br}$ J Cancer 1988; 58: 296-300.

48. Tannock IF. The relation between cell proliferation and the vascular system in a transplanted mouse mammary tumour. Br J Cancer 1968; 22: $258-273$

49. Mallick R, Demmy T. Regional lung chemotherapy techniques. Innovations (Phila) 2011; 6: 1-9.
50. Knierim M, Paku S, Paweletz N, et al. Ultrastructural studies on the lung colonization by nonmetastatic rat tumor cells. Anticancer Res 1986; 6: 669-682.

51. Lapis K, Paku S, Liotta LA. Endothelialization of embolized tumor cells during metastasis formation. Clin Exp Metastasis 1988; 6: 73-89.

52. Paku S, Dome B, Toth R, et al. Organ-specificity of the extravasation process: an ultrastructural study. Clin Exp Metastasis 2000; 18: 481-492.

53. Paku S, Paweletz N, Spiess E, et al. Ultrastructural analysis of experimentally induced invasion in the rat lung by tumor cells metastasizing lymphatically. Anticancer Res 1986; 6: 957-966.

54. Machado EA, Gerard DA, Mitchell JR, et al. Arrest and extravasation of neoplastic cells. An electron microscopy study of serial sections at sequential stages. Virchows Arch A Pathol Anat Histol 1982; 396: 73-89.

\section{SUPPORTING INFORMATION ON THE INTERNET}

The following supporting information may be found in the online version of this article:

Supplementary materials and methods.

Table S1. Primary antibodies and fluorescent dyes used for immunofluorescence.

Figure S1. Tumour cells strip the alveolar epithelium from co-opted vessels.

Figure S2. Connective tissue localization within lung metastasis of HT1080, B16, MAT-B-III, C26, and HT25 cell lines.

Figure S3. Relationship between the metastatic tumor mass and the fine structure of the lungs.

\section{Years ago in the Journal of Pathology...}

The fate of Pseudomonas aeruginosa, Proteus mirabilis and Escherichia coli in the mouse kidney

R. H. Gorrill

Cystic fibrosis and acinar ectasia of the pancreas: Histochemical comparison of the intraluminal secretions

M. N-I. Walters and D. G. A. Gibb

To view these articles, and more, please visit:

www.thejournalofpathology.com

Click 'ALL ISSUES (1892 - 2011)', to read articles going right back to Volume 1, Issue 1.

The Journal of Pathology Understanding Disease
Journal of

The Pathological Society 